\title{
Secondary Alopecia Neoplastica Mimicking Alopecia Areata following Breast Cancer
}

\author{
Efthymia Skafida ${ }^{a}$ Ioanna Triantafyllopoulou ${ }^{b}$ \\ Ioannis Flessas $^{b}$ Michael Liontos $^{a}$ Konstantinos Koutsoukos $^{a}$ \\ Flora Zagouri $^{\text {a }}$ Athanasios-Meletios Dimopoulos ${ }^{a}$ \\ aHaematology-Oncology Unit, Department of Clinical Therapeutics, National and \\ Kapodistrian University of Athens, Alexandra General Hospital, Athens, Greece; ${ }^{b}$ General \\ and Breast Surgery Unit, General and Maternity Hospital "Helena Venizelou," Athens, \\ Greece
}

\author{
Keywords \\ Alopecia neoplastica · Breast cancer $\cdot$ Gastrointestinal metastasis
}

\begin{abstract}
Cutaneous metastases from visceral carcinomas are relatively uncommon, with an overall incidence ranging from 0.7 to $9 \%$. Diagnosis of scalp metastases usually escapes clinicians and dermatologists due to the fact that these metastases are mimicking other benign dermatological conditions. Herein, we present an uncommon case of scalp alopecia neoplastica mimicking alopecia areata due to breast cancer; a 43-year-old woman diagnosed with lobular cancer 3 years previously presented with acute loss of hair in well-circumscribed areas of the scalp and was diagnosed with alopecia areata by a private-practice dermatologist. She was then reevaluated, and due to her history of breast cancer, a biopsy from the scalp was performed and revealed alopecia neoplastica. At the same time that the skin lesions were recognized as disease involvement, the patient presented with dyspepsia, and endoscopy of the upper and lower gastrointestinal tract also revealed metastasis to the stomach and bowel. Gastrointestinal metastasis may occur with several types of cancer, but the stomach and bowel are rare metastatic sites for breast cancer.




\section{Introduction}

Cutaneous metastases from visceral carcinomas are relatively uncommon, with an overall incidence ranging from 0.7 to $9 \%$ [1]. Their clinical appearance is nonspecific, the commonest being cutaneous or subcutaneous nodules of pink or violaceous color [2]. Of note, metastases to the scalp account for less than $4 \%$ of all skin metastases, and they usually present as single or multiple nontender nodules. The primary sites of tumors with scalp metastases are usually the lungs, prostate, and breasts [3]. Diagnosis of scalp metastases usually escapes clinicians and dermatologists due to the fact that these metastases are mimicking other benign dermatological conditions.

Herein, we present an uncommon case of scalp alopecia neoplastica mimicking alopecia areata due to breast cancer.

\section{Case Presentation}

A 43-year-old postmenopausal patient with a breast cancer diagnosis presented to the outpatient clinic of A. Syggros University Hospital for Skin and Venereal Diseases after referral by an oncologist. Three years before, a diagnosis of infiltrative lobular breast cancer, luminal $\mathrm{B}$, had been established. The patient had at the time been diagnosed with cT2N2 breast cancer, whereas CT scans and a bone scan had been normal. However, multiple metastases had been identified on the chest wall, and the patient had been diagnosed with stage IV breast cancer and received chemotherapy with anthracyclines and taxanes for 5 months. Of note, alopecia grade 2 had been recorded, as well as complete remission of skin metastases.

After the therapy, the patient had been able to receive surgery and radiotherapy and remained disease free, as demonstrated in her regular follow-up, for the last 2 years, receiving hormonal treatment with tamoxifen. At that time, the patient had become postmenopausal, and a switch of hormonal treatment had been planned; given the higher risk of recurrence (second to third year after initial diagnosis), a thorough dermatological evaluation had been essential in order to exclude any potential skin metastases. At this point, it is important to note that the patient had already requested dermatological consultation from a privatepractice dermatologist for recent acute loss of hair in well-circumscribed areas of the scalp. The private-practice dermatologist had made the diagnosis of alopecia areata and attributed it to stress.

\section{Dermatological Examination}

On dermatological examination, large areas of the scalp, mainly the parietal and occipital region, presented shiny with significant atrophy submersing from the surrounding skin and

1

Fig. 1. Macroscopic picture of the scalp lesions.

Fig. 2. Macroscopic picture of the scalp lesions.

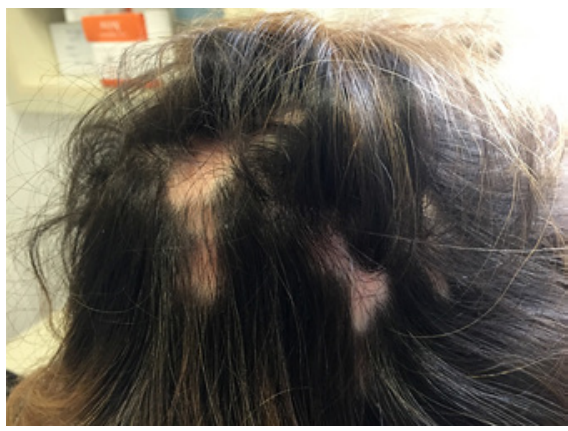

2

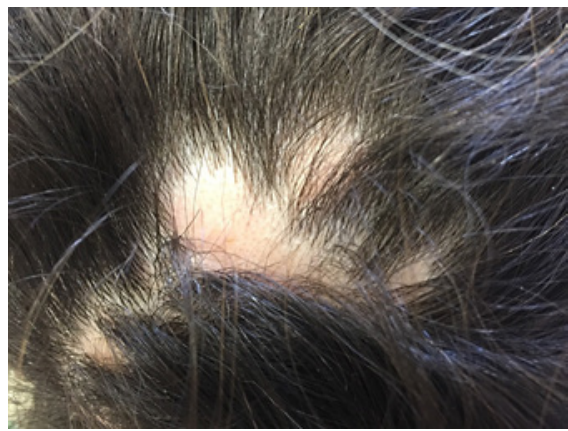




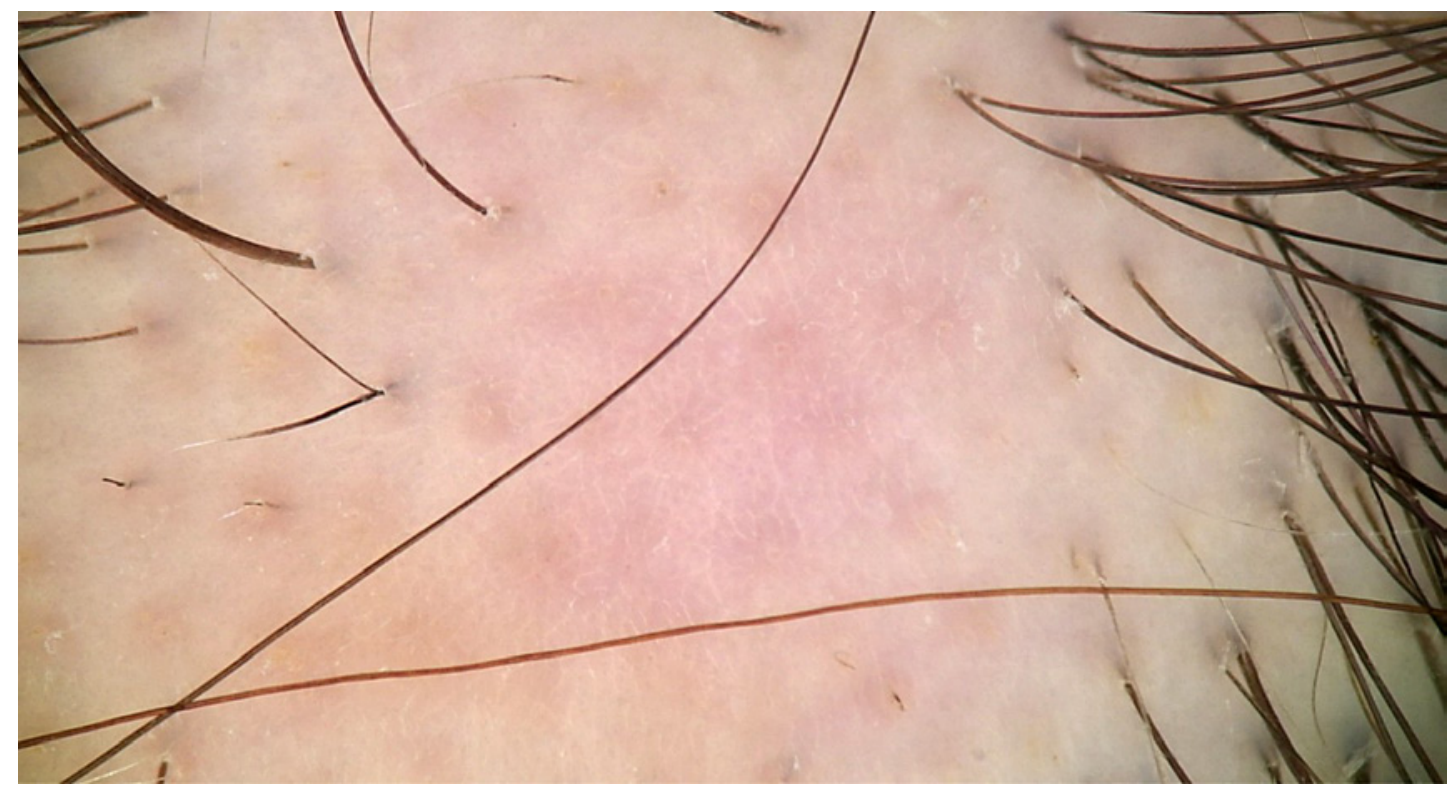

Fig. 3. Macroscopic picture of the scalp lesions.

Fig. 4. Histological examination of the scalp lesion confirming breast cancer metastasis.

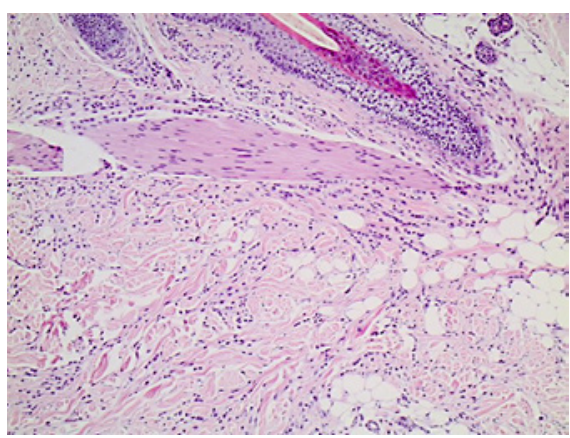

nontender on palpation (Fig. 1, 2). Hair follicles were sparse in these areas. On trichoscopy (Fig. 3), none of the features of alopecia areata could be seen, such as yellow and black dots, exclamation-mark, tapered, broken or vellus hairs, trichorrhexis nodosa, and monilethrixlike hairs.

Another skin finding was the presence of very small, hard-on-palpation, eruptive, subepidermal nodules on the chest wall, neck and face, most probably representing skin metastases, as had been shown on previous histology.

An additional finding was the presence of telangiectatic areas without atrophy on the chest wall, a finding that according to the patient had been existing over the past 17 years, long before the diagnosis of breast cancer. The regional lymph nodes were inconspicuous on palpation. Due to her personal history, a biopsy from the scalp was strongly suggested and performed for alopecia neoplastica.

\section{Histology and Immunochemistry}

The sections showed a diffuse dermal and subcutis infiltration of small cells arranged in cords, as single cells, or around the accessories (Fig. 4). Immunohistochemically, the cells were CK7 (+), E-cadherin(-), ER(+), and PR(+). 


\section{Disease Course}

At the same time that the skin lesions were recognized as disease involvement, the patient presented with dyspepsia; upper gastrointestinal (GI) endoscopy was performed, which revealed two submucosal lesions of the greater curvature, as well as edema and thickening of the rugae in the body and fundus of the stomach. The histological examination confirmed infiltration by metastatic breast cancer cells, with the same molecular profile, i.e., $\operatorname{ER}(+), \operatorname{PR}(+)$, and HER-2(-).

The patient received chemotherapy with 8 cycles of doxorubicin, with significant clinical improvement in both lesions, and continued therapy with fulvestrant. Ten months later, she developed histologically confirmed disease progression in the scalp and switched treatment to exemestane and everolimus. While the patient was asymptomatic, follow-up CT scans 18 months later revealed sigmoid wall thickening, and the patient underwent colonoscopy. The endoscopy and histological examination confirmed metastatic infiltration of the bowel from the lobular breast cancer. At that point, the patient started receiving chemotherapy with eribulin until the present.

\section{Discussion and Conclusions}

In our case, the correct diagnosis of scalp alopecia neoplastica mimicking alopecia areata was a difficult but important step on the patient's evaluation, because it led to reevaluation of her staging and to eventual modification of her treatment. Moreover, this case report underlines the importance of oncologists' collaboration with specialized dermatological centers dealing with adverse events of cancer patients.

Our patient had had skin metastases at the time of diagnosis, and as a result, the potential for recurrence on the skin was higher than that in other breast cancer patients. However, metastases to the scalp are extremely uncommon. To our knowledge, our case is the 5 th ever reported in the literature of alopecia neoplastica mimicking alopecia areata; 3 of the preceding cases were metastases from breast cancer, while the 4th one was from trophoblastic tumor [4-7]. The exact mechanism of alopecia neoplastica is still unknown, as it is unclear whether fibrosis or cytokine secretion from tumor cells leads to disappearance of the hair follicles. According to a recently suggested classification, alopecia neoplastica may be primary or secondary [8]; "primary" refers to all cases of primary tumor arising from the skin and causing local atrophy, whereas "secondary" refers to all metastases to the scalp.

In the majority of patients presenting with alopecia neoplastica, the affected area of the scalp is distinctly erythematous with or without scaling, sometimes covering a subcutaneous nodule or presenting with multiple overlying telangiectases. Severe itching has also been reported, leading to the false diagnosis of eczema. Another clue is the persistence of hair loss in well-defined areas of the scalp after the secondary effects of chemotherapy have subsided. In few cases (17\%), skin metastases occur at the same time or even before the primary cancer has been detected [9].

Concerning therapy for alopecia neoplastica, systemic treatment of the primary tumor is essential. Restoration of alopecia is an option after successful therapy of the primary tumor in cases where scarring has not yet occurred.

In conclusion, it seems that alopecia neoplastica is an uncommon condition, and its diagnosis can easily escape clinicians. This case report underlines the importance of physical examination; despite swift progress in imaging, it seems that physical examination remains the gold standard for screening for early skin metastases. That is why dermatologists should be cautious in the presence of any skin findings, especially in patients with a personal history of cancer. Atypical features of alopecia, refractory to treatment or in patients with underlying malignancies, should raise suspicion of alopecia neoplastica. 
GI metastasis may occur in several types of cancer, with breast cancer being the second most common after lung cancer [10]. However, the stomach and bowel and rare metastatic sites for breast cancer. The incidence of stomach metastasis is about $0.2-1.7 \%$ from all types of cancer and $2-18 \%$ from breast cancer $[10,11]$. Colonic involvement is rarer than gastric involvement, present in 3-12\% of GI tract breast cancer metastatic cases [12]. The histological subtype that most commonly develops GI metastasis is lobular breast cancer. Lobular breast cancer, which constitutes $5-10 \%$ of all breast cancers, more frequently tends to be metastatic to uncommon sites such as the GI tract, ovaries, or peritoneum when compared to ductal carcinoma [13].

Symptoms of GI infiltration may appear years after the first diagnosis and even the therapy of breast cancer. Moreover, as neoplastic cells tend to spare the mucosa, endoscopy is less sensitive for its diagnosis. Primary GI carcinomas also may resemble breast metastasis by radiologic, endoscopic, and histological methods [14, 15]. Thus, diagnosing and distinguishing between them may be challenging but is very important in order to select the best treatment plan for the patients.

Thus, even though staging and routine follow-up endoscopy for women with breast cancer is not recommended, physicians should be aware of this clinical entity, and in case of clinical suspicion, further investigation is warranted.

\section{Statement of Ethics}

Ethical approval for the publication of this case report was obtained from the patient. Written informed consent was obtained from the patient for the publication of this case report including images.

\section{Disclosure Statement}

F. Zagouri has received honoraria for lectures and has served in an advisory role for AstraZeneca, Daiichi, Eli Lilly, Merck, Novartis, Pfizer, and Roche. A.-M. Dimopoulos has received honoraria for participation in advisory boards for Amgen, Bristol Myers Squibb, Celgene, Janssen, and Takeda.

\section{Funding Sources}

The authors declare that they received no funding for this research.

\section{Author Contributions}

All authors contributed to this manuscript and approved the final manuscript.

\section{References}

1 Lookingbill DP, Spangler N, Sexton FM. Skin involvement as the presenting sign of internal carcinoma. A retrospective study of 7,316 cancer patients. J Am Acad Dermatol 1990;22(1);19-26.

2 Krathen RA, Orengo IF, Rosen T. Cutaneous metastasis: a meta-analysis of data. South Med J. 2003 Feb; 96(2):164-7. 
3 Kim HJ, Min HG, Lee ES. Alopecia neoplastica in a patient with gastric carcinoma. Br J Dermatol. 1999 Dec; 141(6):1122-4.

4 Schorr WF, Swanson PM, Gomez F, Reyes CN. Alopecia neoplastica. Hair loss resembling alopecia areata caused by metastatic breast cancer. JAMA. 1970 Aug;213(8):1335-7.

5 Carson HJ, Pellettiere EV, Lack E. Alopecia neoplastica simulating alopecia areata and antedating the detection of primary breast carcinoma. J Cutan Pathol. 1994 Feb;21(1):67-70.

6 Yuen YF, Lewis EJ, Larson JT, Wilke MS, Rest EB, Zachary CB. Scalp metastases mimicking alopecia areata. First case report of placental site trophoblastic tumor presenting as cutaneous metastasis. Dermatol Surg. 1998 May;24(5):587-91.

7 Lin WL, Lin WC, Jung SM, Yang CH, Hong HS. Breast cancer metastasized to the scalp mimicking alopecia areata: alopecia neoplastica. Breast J. 2007 Jan-Feb;13(1):94-5.

8 Cohen PR. Primary alopecia neoplastica versus secondary alopecia neoplastica: a new classification for neoplasm-associated scalp hair loss. J Cutan Pathol. 2009 Aug;36(8):917-8.

9 Cohen PR. Lung cancer-associated scalp hair loss: a rare cause of secondary alopecia neoplastica. Cutis. 2013 Nov;92(5):E7-8.

10 Birla R, Dinu D, Iosif C, Constantinoiu S. Gastric metastasis of invasive lobular breast carcinoma, a current diagnostic and treatment challenge - a review. Chirurgia (Bucur). 2019;114(5):571-8.

11 De Palma GD, Masone S, Rega M, Simeoli I, Donisi M, Addeo P, et al. Metastatic tumors to the stomach: clinical and endoscopic features. World J Gastroenterol. 2006;12(45):7326-8.

12 Galanopoulos M, Gkeros F, Liatsos C, Pontas C, Papaefthymiou A, Viazis N, et al. Secondary metastatic lesions to colon and rectum. Ann Gastroenterol. 2018 May-Jun;31(3):282-7.

13 Mohy-Ud-Din N, Patek B, Dhawan M. Unusual presentation of gastric outlet obstruction due to breast cancer metastasis: a case report. Cureus. 2019 Apr;11(4):e4533.

14 Kim D, Son S, Choi Y. Gastric metastasis from invasive lobular breast cancer, mimicking primary gastric cancer: a case report. Medicine (Baltimore). 2018 Mar;97(13):e0258.

15 Xu L, Liang S, Yan N, Zhang L, Gu H, Fei X, et al. Metastatic gastric cancer from breast carcinoma: a report of 78 cases. Oncol Lett. 2017 Oct;14(4):4069-77. 